\title{
Contingent Skin-Shock Treatment and the Reversal of Effects on Severe Problem Behavior
}

John O'Neill ${ }^{\star}$ and Nathan A. Blenkush

Judge Rotenberg Educational Center, 250 Turnpike St, Canton, MA 02021, United States

\begin{abstract}
We completed a retrospective analysis of the addition and removal of a contingent skin-shock (i.e., positive punishment) procedure as part of the comprehensive behavioral treatment of severe problem behaviors. Participants demonstrated clinically significant decreases in the frequency of severe aggressive and health dangerous (e.g., self-injurious) behaviors when contingent skin-shock was in place. Immediate and substantial increases in problem behavior and emergency restraint were observed upon discontinuation of the procedure. The findings provide support for the supplemental use of contingent skin-shock as the least intrusive, most effective, and efficient treatment available for the severe problem behaviors of some individuals.
\end{abstract}

\section{Publication History:}

Received: June 06, 2020

Accepted: June 28, 2020

Published: June 30, 2020

\section{Keywords:}

Aversive, Aggression, Punishment, Self-injury, Skin-shock

\section{Contingent Skin-Shock Treatment and the Reversal of Effects on Severe Problem Behavior}

Contingent skin-shock (CSS) is a positive punishment procedure that has been subject to experimental analysis in humans for over half a century [1] and has been a source of controversy for behavior analysis [2-4]. In various formats, punishment procedures have been used to treat a range of problem behaviors, typically when there was a risk of serious physical harm, identification and control of maintaining variables could not be achieved, and when treatments derived from functional behavioral assessments had not resulted in clinically significant behavior change. The initial effects of CSS procedures are often superior to the effects of other behavioral procedures (for reviews, see $[5,6]$ ) and are often associated with beneficial side effects such as decreases in non-treated problem behavior, increases in appropriate behavior, and improvements in skill acquisition [7-9].

The use of CSS to treat severe problem behaviors has a substantial evidence base. First, although CSS has unique advantageous properties [10], it is by definition a positive punishment procedure, and thus conceptually supported by associated basic research. That is, the response contingent presentation of stimuli that reduce the frequency of responding has been replicated across a range of organisms, contexts, stimuli, with varying parameters [11]. Punishment (selection by consequence) is integrated into evolutionary theory [12] and various brain mechanisms involved in punishment have been identified [13].

Second, the efficacy of CSS in treating severe problem behaviors has been replicated through various single-subject designs. For example, efficacy has been demonstrated through reversal designs [10-14], multiple baseline designs [15,16], and A-B designs [17]. The effects have been replicated across multiple participants, diagnoses, research groups, devices with varying parameters, behavior functions, and topographies of problem behaviors with durability data ranging from weeks to years $[15,18-20,21]$.

As early as 1988, Iwata offered the opinion that it was clear humans responded to aversive stimuli; by suggesting the following:

Some say that research on punishment with humans has scientific value because it extends similar research conducted with nonhuman subjects. Whom are we kidding? It should be obvious by now that human behavior responds to aversive contingencies, and that we probably do not need to know much more about them in order to develop a generally effective technology of behavior change (p. 151).

However, perhaps because of the controversy surrounding the clinical application of CSS, and misunderstanding regarding singlesubject research designs [22], questions regarding efficacy remain.

For example, in March of 2020, the Food and Drug Administration banned CSS as a treatment for self-injurious and aggressive behavior ${ }^{1}$. The FDA stated the following:

"Although the available data and information show that some individuals subject to ESDs (Electrical Stimulation Devices) exhibit an immediate interruption of the targeted behavior, the available evidence has not established a durable long-term conditioning effect or an overall-favorable benefit-risk profile for the devices" (p.13312).

The purpose of the present analysis was to (a) compare and contrast the comprehensive behavioral treatment with and without a CSS component; (b) further demonstrate the short and long-term effects of adding and abruptly removing the procedure from treatment; and (c) describe the risks and benefits of CSS in two cases.

\section{Method}

The protocol was approved by the Massachusetts Department of Developmental Services Research Review Committee.

${ }^{1}$ The JRC Parents Association and The Judge Rotenberg Center petitioned the FDA for a stay of action in relation to the ban and have filed an appeal of the ban with the United States Court of Appeals for the District of Columbia. The FDA issued a partial stay allowing patients already using ESDs (i.e. the CSS) to continue such use. In the JRC petition for stay of action, a litany of problems with the ban are enumerated and supported by the record and internal FDA documents obtained through Freedom of Information Act.

"Corresponding Author: Dr. John O'Neill, Judge Rotenberg Educational Center 250 Turnpike St, Canton, MA 02021, United States; E-mail: j.oneill@judgerc.org

Citation: O'Neill J, Blenkush NA (2020) Contingent Skin-Shock Treatment and the Reversal of Effects on Severe Problem Behavior. Int J Psychol Behav Anal 6: 168. doi: https://doi.org/10.15344/2455-3867/2020/168

Copyright: (c) 2020 O'Neill et al. This is an open-access article distributed under the terms of the Creative Commons Attribution License, which permits unrestricted use, distribution, and reproduction in any medium, provided the original author and source are credited. 


\section{Participants}

Two individuals participated in the study during their enrollment at the Judge Rotenberg Educational Center (JRC) in Canton, MA. Their guardians provided written informed consent to participate. Both participants were rejected by, unsuccessfully treated in, or expelled from other settings that used a combination of behavioral interventions and psychotropic medications.

Participant 1 was a 16-year-old (upon admission) female diagnosed with intellectual disability (mild), intermittent explosive disorder, fetal alcohol syndrome and psychosis (not otherwise specified). She had a history of aggressive behaviors (hitting, kicking, biting, pinching, throwing objects at others, and homicidal threats) and health dangerous behaviors (suicidal ideation, threats, attempts and auditory hallucinations) that required multiple hospitalizations, among other destructive, disruptive and noncompliant problem behaviors. Upon admission, the psychosis diagnosis was dropped because of absence of associated symptoms. The hypothesized functions of her problem behaviors were escape/avoidance of task demands and attention. Multiple permutations of differential reinforcement procedures were found to be insufficient. She required mechanical restraints (fourpoint board, four-point restraint chair, wrist and ankle cuffs and a helmet with a bite/spit guard) during emergencies. She was able to communicate effectively verbally and required prompts to complete activities of daily living. Prior to admission, she was prescribed topiramate $(600 \mathrm{mg} /$ day $)$, quetiapine $(300 \mathrm{mg} /$ day), ziprasidone (160 $\mathrm{mg} /$ day) and chlorpromazine $(50 \mathrm{mg} /$ day) to treat problem behaviors. Upon admission, she required 24 hour/day supervision to maintain her safety.

Participant 2 was a 10-year-old (upon admission) male diagnosed with intellectual disability (severe) and autism spectrum disorder (Level III)). He had a history of health dangerous behaviors (selfinjurious biting to the point of drawing blood, bruxism, fecal smearing, elopement) and aggressive behaviors (biting, hitting, kicking, pushing, pinching, scratching, forcefully grabbing and pulling the hair others), among other destructive, disruptive and noncompliant problem behaviors. He required acute hospitalization on multiple occasions because of the intensity of his self-injury. The hypothesized function of his aggression was multiple maintaining factors (escape/ avoidance, attention and tangible access). The hypothesized function of his health dangerous behavior was automatic (unknown). Multiple permutations of differential reinforcement procedures were found to be insufficient. He wore arm splints throughout the day to prevent him from injuring his hands and required mechanical restraint in vehicles because of physical aggression towards peers and direct care staff. He used a picture exchange communication system to communicate, was not toilet-trained and required hand-over-hand prompting to complete activities of daily living. Prior to admission, he was prescribed risperidone to treat problem behaviors. Cogentin, clonidine, valproic acid, haloperidol and sertraline were also prescribed in his past. Upon admission, he was assigned a 24 hour/ day 1:1 paraprofessional to supervise and maintain his safety.

\section{Setting}

Both participants were cared for by JRC's day program (i.e., school and workshop) and residential facilities (i.e., community apartments and houses) where they received behavioral treatment, education, and development opportunities year-round. A doctoral level clinician, with a typical caseload of 15 to 20 , oversaw each participant's comprehensive behavioral treatment with the assistance of other members of the treatment team, such as the case manager, teacher, residential supervisor and direct care staff. Consistent treatment procedures were in place and implemented in the day program buildings, residences, on field trips, and during transportation. The participants' treatment in both day and residential settings were monitored directly by on-scene supervisors and remotely by supervisors who watched live and videorecorded footage across facilities. Observations were completed on a time-sampling or continuous basis, depending on client needs. Video cameras and microphones were mounted in appropriate locations of the school and residential halls, rooms, and exterior perimeter. This equipment allowed the supervisors to monitor from a central office, in real-time, all activities in the participants' classrooms and residences from multiple angles.

\section{Apparatus}

CSS was administered via the Graduated Electronic Decelerator (GED). See Israel, Blenkush, von Heyn, and Rivera [24] for a technical description of the GED components. Blenkush and O'Neill [17] provided a comparison with the electrical parameters of other devices.

\section{Safeguards}

The following safeguards were in effect prior to the use of CSS: (1) The parent/guardian provided written informed consent to the use of CSS that could be revoked at any time. (2) If the participant was of school age, CSS was placed in his or her Individualized Education Plan. If the participant was an adult, CSS was placed in his or her Individualized Service Plan. (3) A doctoral level clinician, with training in behavioral psychology, headed the participant's treatment team and composed a treatment plan that included the option to employ CSS. (4) A physician and, where appropriate, a neurologist and/or cardiologist certified the absence of medical contraindications to the use of CSS. (5) A psychiatrist certified the absence of psychiatric contraindications to the use of CSS for each participant who had a mental illness diagnosis. (6) A peer-review committee, typically comprised of JRC clinicians and outside members, assessed the treatment plan and deemed it appropriate. (7) A human rights committee, composed of JRC parents and community members unaffiliated with JRC, assessed and approved the treatment plan. (8) A Massachusetts Probate Court judge authorized the treatment plan through a "substituted judgment" petition in an individual court hearing in which the participant was represented by his or her own court-appointed attorney. (9) The court-appointed attorney retained his or her own psychologist or psychiatrist to provide advice concerning the appropriateness of the proposed treatment plan. The court-appointed attorney had the opportunity to object to any aspect of the treatment plan before and during the implementation of the plan. (10) All staff that administered CSS were trained on the relevant policies and procedures (see Procedures section) on an annual basis.

Additional safeguards were in effect after court-approval of the treatment plan. Staff members who administered CSS completed competency-based training regarding CSS administration and were prompted to report any adverse effect associated with the treatment. Reports on the participant's treatment status (including the frequency of GED applications) were submitted to the Probate Court every 3 months and the judge held a formal review each year at a minimum. In all cases in which CSS was used for 3 or more years, a special committee composed of staff and consultants, including two independent psychologists unaffiliated with JRC, reviewed the treatment plan and its results to determine if it should continue. 


\section{Target behaviors and data collection}

For Participant 1, aggressive behavior was defined as hit others, push others, scratch others, forcefully grab others, kick others, and bite others. Health dangerous behavior was defined as bite self, hit self, scratch self, bang head, throw self to the floor, forceful head movements including whipping neck forward and backward, knee self in face, and pull hair out.

For Participant 2, aggressive behavior was defined as hit others, bite others, kick others, pinch others, and throw objects at others, including all attempts. Health dangerous behavior was defined as any attempt to injure himself, bite self, hit self, smear feces on self, slap face/legs, clap/slap hands forcefully to cause redness, eat inedible objects.

For the first participant, other categories of behavior were also treated with CSS such as destructive (e.g., break object), noncompliant (e.g., refuse to follow a learned direction during an emergency), and major disruptive (e.g. scream, spitting on others).Because participants differed in whether or not these behaviors were treated with CSS, behavior frequencies associated with these categories were not included in the data. However, the frequency of CSS applications, regardless of behavior category, is reported.

Behavioral observations and data collection were conducted around the clock by school, day program, and residential staff including direct-care, teachers, training, programming, clinical, and administrative staff. Frequency per hour was recorded by placing a tally in the appropriate cell on a recording sheet each time a client engaged in an operationally defined behavior. Every application of CSS for targeted behaviors was recorded in a similar manner to behaviors and was verified, before application, by a second individual who was trained on relevant policies and procedures. Emergency restraint forms were utilized to record the time in and out for every instance. A charting department entered the data and conducted regular integrity checks. A data management system enabled the participant's clinician to review daily behavioral data for level, trend, and variability across days, weeks, and months to assess the effects of interventions.

\section{Design}

A concurrent multiple-baseline was combined with a reversal design (i.e., $\mathrm{ABAB}$ ) to display and analyze the effect of adding and removing CSS from participant's comprehensive behavioral treatment. CSS was removed because either the attending clinician chose to dispense with the treatment based on treatment factors or because of regulatory changes pertaining to CSS use. CSS was reinstated either because of legal victories that once again made CSS available or clinical decisions based on observed increases in problem behaviors.

\section{Procedure}

Upon admission, functional behavior assessments were completed for each participant. To account for the various possible functions, all environments and staff procedures were designed to minimize inadvertent or deliberate reinforcement of problem behaviors. In particular, staff were trained to ensure that (1) positive/negative attention would be minimized or avoided whenever problem behaviors occurred; (2) escape/avoidance from demands was minimized; and (3) potential reinforcing tangible items or activities were not available following problem behavior.

Int J Psychol Behav Anal

ISSN: 2456-3501

\section{Non-CSS phase (A)}

Both participant's treatment included preference assessments, antecedent/environmental manipulations, differential reinforcement of alternative (DRA), incompatible (DRI), and other (DRO) behaviors, functional communication training, extinction, response cost, and other behavioral procedures that, in sum, amounted to an ongoing functional analysis. All skills were taught through prompt hierarchies, shaping, and redirection to appropriate behaviors. Participant 1 was capable of rule-governed behavior and received behavioral counseling and self-management training. Differential reinforcement procedures utilized with her spanned minutes to days. Participant 2 received 1:1 staffing and wore arm splints to prevent hand biting. Differential reinforcement procedures utilized with him spanned seconds to minutes and focused on basic skills such as sitting and following simple directions.

\section{CSS phase (B)}

In this phase, all the behavioral procedures employed during the non-CSS phase continued to be used and adjusted by the clinicians. However, CSS applications were administered as soon as possible following all topographies listed under the aggression and health dangerous categories. The procedure for administering a CSS application required the staff member to enlist a second staff member to ensure (1) selection of the correct recording sheet for the participant, (2) the topography that occurred was listed on the recording sheet as a treatment target, (3) the consequence (i.e., CSS) was specified for said topography, (4) selection of the correct remote control for the participant, and (5) application of the CSS. These requirements introduced a short delay between the occurrence of a target behavior and the administration of the consequence but ensured treatment integrity.

Each time the participant engaged in a target behavior, the staff member who administered the CSS recorded the apparent antecedent stimulus as well as other potential setting event information on the participant's daily recording sheet. This information was used by the clinician, in conjunction with direct observations, as part of their ongoing functional analysis of the problem behaviors.

\section{Results}

Figure 1 displays the number of problem behaviors (i.e., combined aggressive and health dangerous behaviors) per month for each participant during non-CSS treatment (A) and CSS treatment (B) phases. Both participants were successfully tapered off all psychotropic medications by their attending psychiatrist during the first three months of non-CSS treatment and continued medication-free for the remainder of the study.

\section{Participant 1}

During the initial 9 month non-CSS phase, problem behaviors ( $M=590$, range 334-1059) were observed at high, variable monthly rates, with a slight decreasing trend. During the first full month of CSS treatment, a 99\% reduction was observed in the monthly rates of problem behaviors. In the months $(n=18)$ following the onset of CSS treatment, problem behaviors ( $M=3$, range 0-13) maintained at low, stable monthly rates, with stability in the number of CSS applications per month $(M=6$, range $0-44)$. During the first month of CSS treatment removal, an immediate and drastic increase was observed in 
Citation: O’Neill J, Blenkush NA (2020) Contingent Skin-Shock Treatment and the Reversal of Effects on Severe Problem Behavior. Int J Psychol Behav Anal 6: 168. doi: https://doi.org/10.15344/2455-3867/2020/168

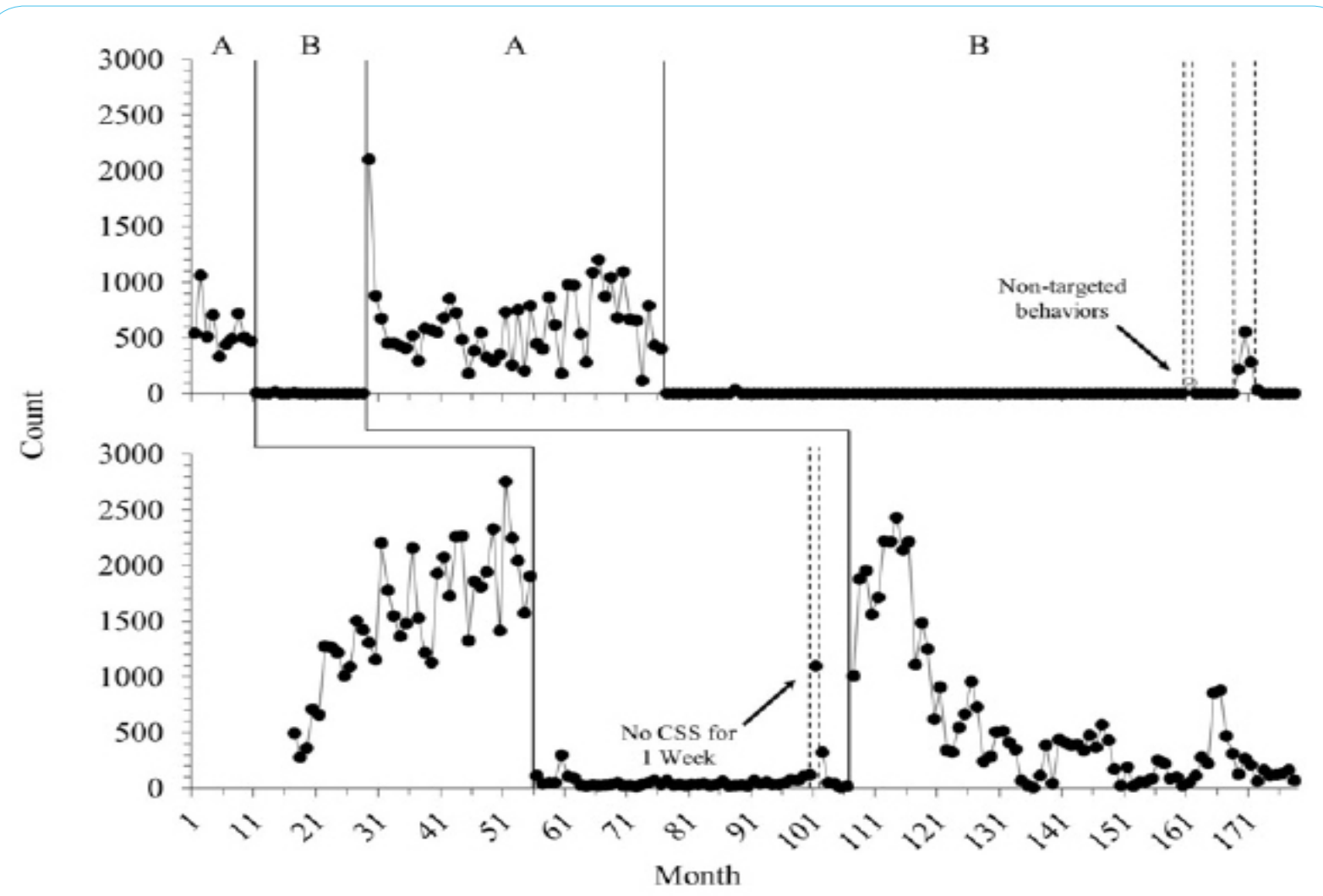

Figure 1: The number of severe problem behaviors per month for Participant 1 (top) and Participant 2 (bottom) during differential reinforcement without contingent skin-shock treatment $(\mathrm{A})$ and differential reinforcement with contingent skin-shock treatment (B). Dashed vertical lines indicate brief treatment fading attempts.

the monthly rate of problem behaviors, far beyond the level observed during the initial non-CSS phase. In the months $(n=46)$ following the removal of CSS treatment, problem behaviors $(M=593$, range 1151202) were observed at high, variable monthly rates, with a relatively stable trend. In the months $(n=83)$ following the reinstatement of CSS treatment, problem behaviors $(M=0$, range $0-32)$ were again observed at low, stable monthly rates, with stability in the number of CSS applications per month $(M=0$, range 0-3). Subsequently, two fading attempts were conducted but resulted in immediate and drastic increases in non-targeted and targeted problem behaviors $(M$ $=352$, range 217-556), respectively. Following a final reinstatement of CSS treatment, problem behaviors were reduced to zero and maintained with zero CSS applications across months. In total, 133 CSS applications were administered and equated to approximately 4 min of aversive stimulation across the course of 12 years.

\section{Participant 2}

During the initial 38 month non-CSS phase, problem behaviors ( $M=1519$, range 281-2756) were observed at high, variable monthly rates, with an increasing trend. During the first full month of CSS treatment, a $92 \%$ reduction was observed in the monthly rates of problem behaviors. In the months $(n=45)$ following the onset of CSS treatment, problem behaviors $(M=53$, range 14-296) maintained at low, stable monthly rates, with variability in the number of CSS applications per month $(M=34$, range 6-102). A 1 week fading attempt resulted in an immediate and drastic increase in problem behaviors. In the months $(n=71)$ following the removal of CSS treatment, problem behaviors $(M=562$, range 7-2434) were observed at high, variable monthly rates, with a decreasing trend.

\section{Collateral restraint effect}

Table 1 displays the total number and duration of physical restraints during each phase of treatment for both participants. As compared to non-CSS phases (A), CSS phases (B) were associated with substantial decreases in the number of emergency restraints $(M=90 \%$, range 88 $94 \%)$ and total duration spent in restraint $(M=94 \%$, range $87-98 \%)$.

\begin{tabular}{|l|l|l|l|l|}
\hline \multicolumn{5}{|c|}{ Number of Emergency Restraints (mins) } \\
\hline Participant & A & B & A & B \\
\hline 1 & $215(2170)$ & $13(35)$ & $359(4880)$ & $44(653)$ \\
\hline 2 & $302(5543)$ & $32(238)$ & $319(2039)$ & - \\
\hline
\end{tabular}

Table 1: Collateral Restraint Effects of Contingent Skin-Shock.

The total number and duration (min) of emergency restraints during differential reinforcement without contingent skin-shock treatment (A) and differential reinforcement with contingent skin-shock treatment (B) for each participant.

\section{Discussion}

The results demonstrate the effect of adding and abruptly removing CSS from the comprehensive behavioral treatment of severe problem behaviors demonstrated by two individuals with intellectual disability. In total, the analyses contain approximately 28 years of clinical data. The addition of CSS to treatment resulted in clinically significant reductions in aggressive and health dangerous behaviors during the first full month of CSS treatment, followed by maintenance of the effect for years. This result is in line with the most recent literature review on the use of punishment procedures in clinical settings and 
Citation: O’Neill J, Blenkush NA (2020) Contingent Skin-Shock Treatment and the Reversal of Effects on Severe Problem Behavior. Int J Psychol Behav Anal 6: 168. doi: https://doi.org/10.15344/2455-3867/2020/168

Page 5 of 5

provides support for the assertions that (a) punishment procedures may be necessary when control of the problem behavior's maintaining variables is not possible, (b) punishment may be preferable to reinforcement-based procedures when there is a risk of serious physical harm, and (c) treatments derived from functional analyses (e.g., DRA) may not always reduce behavior to a clinically acceptable level without a punishment component [5].

In the present cases, removal of CSS treatment was mandated by a state regulatory body and resulted in immediate and drastic increases in the level, trend, and variability of problem behaviors. Associated increases were also observed in the number and duration of emergency restraints. In fact, the problem behaviors of one participant did not consistently return to the low level and invariability observed during the CSS phase, even after years of comprehensive behavioral treatment. These findings provide further support for the assertion that any fading of CSS treatment should be systematically planned by the attending clinician [17].

Emergency restraint was significantly curtailed for both participants and arm splints were not needed for Participant 2 with CSS in effect. Thus, the introduction of CSS reduced the overall restrictiveness of treatment. The duration of aversive stimulation applied during emergency restraint procedures far outweighed that of the CSS procedure for both participants. Counterintuitively, this finding suggests that CSS procedures might actually reduce the overall level of aversive stimulation for individuals with severe problem behaviors.

The reversal effect replicates the effect of CSS. However, the return of the problem behavior to baseline also suggests that CSS may be prosthetic in nature for some patients. Like many behavioral treatments (e.g. extinction, differential reinforcement, and antecedent interventions), psychopharmacological treatments, and protective equipment, termination of the treatment results in a return of the problem behaviors. Severe behavior disorders are typically chronic conditions and require long-term management. Effective, yet noncurative treatments, are valuable given that many patients are ineffectively treated for years or decades.

\section{Limitations}

Due to the clinical nature of the study, experimental control was not absolute. The data represent an analysis of CSS as part of a comprehensive behavioral treatment plan that included various other overlapping procedures. Although staff who collected the data received a substantial amount of training and were monitored via digital video recording to maintain treatment integrity, the frequency data lacks a direct measure of inter-observer reliability.

\section{Conclusion}

Severe problem behaviors present substantial risk of harm to the individual and others. Comprehensive behavioral treatment with a supplemental CSS procedure is more effective, efficient, and ethically responsible than the continuation of treatments that are ineffective, unlikely to confer immediate benefit, or do not substantially reduce the risk of harm.

\section{Competing Interests}

The authors declare that they have no competing interests.

\section{References}

1. Lovaas OI, Schaeffer B, Simmons JQ (1965) Building social behavior in autistic children by use of electric shock. Journal of Experimental Research in Personality 1: 99-109.

2. Iwata BA (1988) The development and adoption of controversial default technologies. Behav Anal 11: 149-157.

3. Johnston JM (1991) What can behavior analysis learn from the aversives controversy? The Behav Anal 14: 187-196.

4. Sherman RA (1991) Aversives, fundamental rights and the courts. Behav Anal 14: 197-206.

5. Lerman DC, Vorndran CM (2002) On the status of knowledge for using punishment: Implications for treating behavior disorders. J Appl Behav Anal 35: 431-464.

6. Carr EG, Lovaas OI (1983) Contingent electric shock as a treatment for severe behavior problems. In S Axelrod \& J Apsche (Eds.). The effects of punishment on human behavior. New York: Academic Press.

7. Blenkush NA (2017) A risk-benefit analysis of antipsychotic medication and contingent skin shock for the treatment of destructive behaviors. International Journal of Psychology \& Behavior Analysis 3: 121.

8. Matson J, Taras M (1989) A 20 year review of punishment and alternative methods to treat problem behaviors in developmentally delayed persons. Res Dev Disabil 10: 85-104.

9. van Oorsouw WMWJ, Israel ML, von Heyn RE, Duker PC (2008) Side effects of contingent shock treatment. Res Dev Disabil 29: 513-523.

10. Linscheid TR, Iwata BA, Ricketts RW, Williams DE, Griffin JC (1990) Clinical evaluation of the self-injurious behavior inhibiting system (SIBIS). J Appl Behav Anal 23: 53-78.

11. Azrin NH, Holz WC (1966) Punishment. In WK Honig (Ed.), Operant behavior: Areas of research and application. New York: Appleton-Century-Crofts.

12. Shah MJ, Commons ML (2019) A developmental and evolutionary theory of punishment. Ethics, Medicine and Public Health 8: 108-119.

13. Jean-Richard-Dit-Bressel P, Killcross S, McNally GP (2018) Behavioral and neurobiological mechanisms of punishment: implications for psychiatric disorders. Neuropsychopharmacology 43: 1639-1650.

14. Salvy SJ, Mulick JA, Butter E, Bartlett RK, Linscheid TR (2004) Contingent Electric Shock (SIBIS) and a Conditioned Punisher Eliminate Severe Head Banging in a Preschool Child. Behavioral Interventions 19: 59-72.

15. Blenkush NA, O'Neill J (2020) Contingent skin-shock treatment in 173 cases of severe problem behavior. Int J Psychol Behav Anal 6: 167.

16. Mudford OC, Boundy K, Murray AD (1995) Therapeutic Shock Device (TSD): Clinical Evaluation With Self-Injurious Behaviors. Res Dev Disabil 16: 253267.

17. Israel ML, Blenkush NA, von Heyn RE, Sands CC (2010) Seven case studies of individuals expelled from positive-only programs. The Journal of Behavior Analysis of Offender and Victim Treatment and Prevention 2: 20-36.

18. Whaley D L, Tough J (1968) Treatment of a self-injuring mongoloid withshock-induced suppression and avoidance. State of Michigan, Department of Mental Health Research Bulletin 2: 33-35.

19. Lovaas OI, Simmons JQ (1969) Manipulation of self-destruction in three retarded children. J Appl Behav Anal 2: 143-157.

20. Duker PC, Seys DM (1996) Long-term use of electrical aversion treatment with self-injurious behavior. Res Dev Disabil 17: 293-301.

21. Israel ML, Blenkush NA, von Heyn RE, Rivera PM (2008) Treatment of aggression with behavioral programming that includes supplementary skin shock. The Journal of Behavior Analysis of Offender and Victim Treatment and Prevention 1: 119-166.

22. Dermer ML, Hoch TA (1999) Improving descriptions of single-subject experiments in research texts written for undergraduates. The Psychological Record 49: 49-66. 